\title{
Analysis and Solution of Abnormal Fault of Data Storage Based on NAND-flash
}

\author{
Zhao Long*, Shen Xiaohe, Chen Geng, Hu Xiaoxi \\ Beijing Institute of Aerospace Control Devices, Beijing, China \\ Email address: \\ 15611796238@163.com (Zhao Long), shenxiaohe2008@163.com (Shen Xiaohe), chengeng191@163.com (Chen Geng), \\ Huxx615@126.com (Hu Xiaoxi) \\ ${ }^{*}$ Corresponding author
}

\section{To cite this article:}

Zhao Long, Shen Xiaohe, Chen Geng, Hu Xiaoxi. Analysis and Solution of Abnormal Fault of Data Storage Based on NAND-flash. Science Discovery. Vol. 8, No. 4, 2020, pp. 69-73. doi: 10.11648/j.sd.20200804.12

Received: June 18, 2020; Accepted: August 18, 2020; Published: August 25, 2020

\begin{abstract}
NAND-flash memory has the advantages of large capacity and fast rewriting speed. It is suitable for the storage of large amounts of data and is often used as an online storage device for embedded products. However, NAND-flash has the problems of bad blocks and other insufficient reliability.In a certain aerospace model temperature/strain measurement system, the NAND-flash memory is erased and written through DSP software, and the strain/temperature measurement data collected by the sensor is stored in real time. After the system was powered on many times and completed data collection and decoding, it was found that the data stored in the NAND-flash had abnormal faults. By analyzing the test phenomenon and failure mechanism, the failure problem is attributed to the fact that the data in the NAND-flash is not erased, and it is coupled with the newly written data, and it is finally located because the data in the original data address cannot be effectively erased when the data address is stored. Coupling also occurred, resulting in an error in the data address, and the data was coupled after power-on again. Based on the above-mentioned reasons, this paper proposed a troubleshooting method and conducted a test. The verification was successfully passed and the problem was resolved.This method has high reference significance in the large-capacity and high-reliability data storage of NAND-flash in the field of aerospace models.
\end{abstract}

Keywords: NAND-flash, Data Exception, Fault Analysis

\section{基于NAND-flash的数据存储异常故障分析及解决方法}

赵龙 ${ }^{*}$ ，小禾，陈费，胡晓曦

北京航天控制仪器研究所, 北京, 中国

邮箱

15611796238@163.com（赵龙）, shenxiaohe2008@163.com（申小禾）, chengeng191@163.com（陈春）, Huxx615@126.com（胡晓曦）

摘要: NAND-flash存储器具有容量较大、改写速度快等优点, 适用于大量数据的存储, 经常作为嵌入式产品的线上存 储设备, 但NAND-flash存在坏块及其他可靠性不足的问题。某航天型号温度/应变测量系统中, 通过DSP软件对 NAND-flash存储器进行擦除、写入等操作, 实时存储传感器采集得到的应变/温度测量数据。在系统多次上电并完成数 据采集和解码后, 发现NAND-flash中存储的数据存在异常故障。通过分析试验现象和故障机理, 将故障问题归结为 NAND-flash中数据未擦除, 与新写入数据发生耦合, 最终定位于由于储存数据地址时未能有效擦除原先数据地址中的 数据, 也发生了耦合, 导致数据地址出错, 从而重新上电后数据耦合, 根据上述原因本文提出了故障解决方法并及进 行了试验, 顺利通过了验证, 问题得到解决。该方法在航天型号领域NAND-flash的大容量、高可靠性数据存储方面具 有较高的参考意义。 
关键词: NAND-flash, 数据异常, 故障分析

\section{1. 引言}

某航天型号温度/应变测量系统中, 通过采用光纤光 栅传感器, 能够同时完成96路以上温度/应变的实时测量, 在测量过程中产生了大量的数据, 需要一种能够快速可靠 的存储方案保证数据的正确性和完整性。在系统设计时, 采用NAND-flash作为数据的存储器。针对NAND-flash使 用的实践可靠性, 经过研究发现, NAND-flash在使用过程 中存在坏块、擦除失败等可靠性不足的问题, 众多研究人 员提出了针对此类问题的可靠性设计方法和措施, 解决了 部分可靠性问题，但在实际使用过程中仍然存在不够全面， 问题覆盖性不足的问题 [1-4]。在该航天型号系统产品设计 师, 集合了上述研究成果及设计思路, 但进在行系统测试 时, 通过多次上电并完成数据采集和解码, 并对数据进行 分析后发现, 部分数据仍出现乱码的情况, 且该情况出现 具有一定的概率性和偶然性, 出现的时机为上电后的一段 时间, 往后数据即恢复正常。因数据异常导致温度/应变 测量结果的不正确性, 会导致总体和设计师误判系统的安 全性和可靠性。因此, 分析NAND-flash中存储的数据异常 故障的产生机理并采取有效措施实现对系统温度/应变的 正确测量在实际中有着重要意义。

\section{2. 数据存储的可靠性设计}

Flash闪存是非失易存储器, 可以对称为块的存储器单 元块进行擦写和再编程[5]。任何flash器件的写入操作只能 在空或已擦除的单元内进行, 所以大多数情况下, 在进行
写入操作之前必须先执行擦除。擦除NAND器件是以 $8 \sim 32 \mathrm{~KB}$ 的块进行的, 执行相同的操作最多只需要 $4 \mathrm{~ms}$, 而 擦除NOR器件时是以 $64 \sim 128 \mathrm{~KB}$ 的块进行的, 执行一个写 入/擦除操作的时间为 $5 \mathrm{~s}$, 所以一般在数据存储领域采用 NAND-flash[6]。NAND-flash存储器是flash存储器的一种, 其内部采用非线性宏单元模式, 为固态大容量内存的实现 提供了廉价有效的解决方案。NAND-flash存储器具有容量 较大、改写速度快等优点, 适用于大量数据的存储, 因而 在业界得到了越来越广泛的应用, 如嵌入式产品中, 包括 数据相机、MP3随身听记忆卡、体积小巧的U盘等[7] [12]。 在某航天型号温度/应变测量系统的设计和软件编程过程 中, 开展了一系列数据存储的可靠性设计, 主要采取以下 措施和方案。

\section{1. 坏块的可靠性处理}

NAND-flash非常容易出现坏块, 且NAND器件中的坏 块是随机分布的, 需要对介质进行初始化扫描以发现坏块, 并将坏块标记为不可用。针对NAND-flash中的坏块, 通过 对全盘进行擦除过程中, 进行有效性校验和检测, 如存在 坏块情况即将坏块地址存储于flash的某固定区域, 在下一 次上电初始化时直接读取该固定区域获得全部的坏块地 址, 在进行数据存储时, 判断是否为坏块, 如果是则跳过, 不将数据存入该坏块中, 以此保证数据存储的可靠性。具 体技术实现如图1所示, 坏块badpage1和坏块badpage2的地 址分别存储于 badpageaddr中, 在系统上电启动时, 从 badpageaddr中读取坏块的地址, 从而在数据存储过程中将 其跳过。

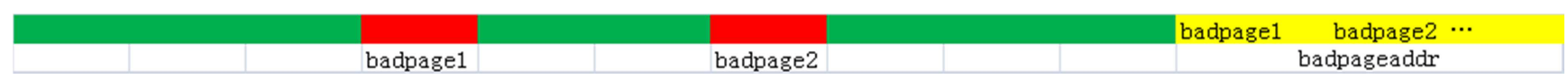

图1 坏块可靠性处理示意图。

\section{2. 写入操作的可靠性设计}

NAND-flash以块为单位进行擦除操作。Flash的写入 操作必须在空白区域进行, 如果目标区域已经有数据, 必 须先擦除后写入, 因此擦除操作是闪存的基本操作。该系 统通过擦除指令, 能够保证全盘的一次性擦除, 同时, 对 是否擦除成功进行了校验, 保证擦除的完整性 [13]。

\section{3. 多次上电的可靠性设计}

因系统需要多次上电使用, 为了保证上一次上电的数 据不被本次上电存储的数据破坏, 系统采用了双地址交叉
存储上一次上电已到达NAND-flash的块地址, 在系统上电 后, 读取该地址确定目前已存储的块, 同时, 因存储的块 地址有一定的延后性, 在读取该地址后自动隔离一个存储 周期的地址长度, 作为本次上电的初始块地址, 以保证上 一次最后的块地址和本次初始块地址不存在交叉的情况。 具体技术实现如图2所示, 系统上电后从块地址存储区中 读取交叉存储的已到达NAND-flash的块地址, 比较addr1 和addr 2 的大小, 取较大值, 并加上一个存储周期的地址 长度, 作为下一次上电的初始块地址。

\begin{tabular}{|c|c|c|c|c|}
\hline 上电后采集的温度/应变数据 & addr1 & 上电初始地址 & addr2 \\
\hline
\end{tabular}

图2 多次上电的可靠性设计。 


\section{3. 故障现象和原因分析}

\section{1. 故障现象}

温度/应变测量系统多次上电并完成数据采集和解码, 并对数据进行分析后发现, 部分数据出现乱码的情况, 且
该情况出现具有一定的概率性和偶然性, 出现的时机为上 电后的一段时间, 往后数据即恢复正常。上电初始异常数 据见图3, 因上电后时间尚未同步, 故输出时间应为初始 化时间（2019-1-10:0:0），但输出数据时间显示为 （2019-0-00:0:0），数据异常。

\begin{tabular}{|c|c|}
\hline 31388 & $2019-12-6 \quad 13: 47: 36$ \\
\hline 31389 & $2019-12-6 \quad 13: 47: 36$ \\
\hline 31390 & $2019-12-6 \quad 13: 47: 37$ \\
\hline 31391 & $2019-12-6 \quad 13: 47: 37$ \\
\hline 31392 & $2019-0-0 \quad 0: 0: 0$ \\
\hline 31393 & $2019-0-0 \quad 0: 0: 0$ \\
\hline 31394 & $2019-0-0 \quad 0: 0: 0$ \\
\hline 31395 & $2019-0-0 \quad 0: 0: 0$ \\
\hline 31396 & $2019-0-0 \quad 0: 0: 0$ \\
\hline 31397 & $2019-0-0 \quad 0: 0: 0$ \\
\hline 31398 & $2019-0-0 \quad 0: 0: 0$ \\
\hline 31399 & $2019-0-0 \quad 0: 0: 0$ \\
\hline 31400 & $2019-0-0 \quad 0: 0: 0$ \\
\hline
\end{tabular}

31400 2019-0-0 0:0:0

\begin{tabular}{|r|r|r|}
\hline 932 & 229597 & 30.4375 \\
\hline 982 & 229598 & 30.4375 \\
\hline 32 & 229599 & 30.4375 \\
\hline 82 & 229600 & 30.4375 \\
\hline 288 & 1 & 20 \\
\hline 346 & 2 & 20 \\
\hline 384 & 3 & 20 \\
\hline 386 & 4 & 20 \\
\hline 496 & 5 & 20 \\
\hline 546 & 6 & 20 \\
\hline 592 & 7 & 20 \\
\hline 642 & 8 & 20 \\
\hline 696 & 9 & 26.1875 \\
\hline
\end{tabular}

30. 75 CF01F81 CH1: 30. $7511401 \mathrm{~F} 81 \mathrm{CH} 1$ : 30. $7515901 \mathrm{~F} 81 \mathrm{CH} 1$ : 30. 75 19E01F81 CH1: $202081 \mathrm{CH} 1$ : $204502081 \mathrm{CH} 1$ : $208 \mathrm{~A} 02081 \mathrm{CH} 1$ : $20 \mathrm{CF} 02081 \mathrm{CH} 1$ $2011402081 \mathrm{CH} 1$ : $2015902081 \mathrm{CH} 1$ : 20 19E02081 CH1:

26. $252181 \mathrm{CH} 1$

26. $254502181 \mathrm{CH} 1$ : \begin{tabular}{ll|l|l|l|l|l|l|l|l|l|}
1533.655 & 1540.7 & 1547.982 & 1554.652 & 1559.421 & 1564.631
\end{tabular} \begin{tabular}{l|r|r|r|r|r|r}
1533.654 & 1540.701 & 1547.982 & 1554.652 & 1559.421 & 1564.631
\end{tabular} \begin{tabular}{lll|l|l|l|l|l}
1533.654 & 1540.7 & 1547.982 & 1554.652 & 1559.421 & 1564.632
\end{tabular} $\begin{array}{llllllll}1533.654 & 1540.7 & 1547.982 & 1554.653 & 1559.421 & 1564.632\end{array}$

\begin{tabular}{|l|l|l|l|l|l|}
\hline 1525 & 1525 & 1525 & 1525 & 1525 & 1525 \\
\hline 1525 & 1525 & 1525 & 1525 & 1525 & 1525 \\
\hline 1525 & 1525 & 1525 & 1525 & 1525 & 1525 \\
\hline 1525 & 1525 & 1525 & 1525 & 1525 & 1525 \\
\hline 1525 & 1525 & 1525 & 1525 & 1525 & 1525 \\
\hline 1525 & 1525 & 1525 & 1525 & 1525 & 1525 \\
\hline 1525 & 1525 & 1525 & 1525 & 1525 & 1525 \\
\hline 1525 & 1525 & 1525 & 1525 & 1525 & 1525 \\
\hline
\end{tabular}

图3 上电初始异常数据。

上电一段时间后，数据恢复正常，具体见图4所示，数据恢复正常过程中出现了数据的异常跳动。

\begin{tabular}{|c|c|c|c|c|c|c|c|c|c|c|c|c|}
\hline & & & & & & & & & & & & \\
\hline 36085 & $2019-12-6 \quad 9: 36: 43$ & 142 & 4630 & 26.3125 & 26. $25 \mathrm{CF} 0 \mathrm{BE} 83$ & CH1: & 1525 & 1525 & 1525 & 1525 & 1525 & 1525 \\
\hline 36086 & $2019-12-6 \quad 9: 36: 43$ & 248 & 4631 & 26. 3125 & 26. $251140 \mathrm{BE} 83$ & CH1: & 1525 & 1525 & 1525 & 1525 & 1525 & 1525 \\
\hline 36087 & $2019-12-6 \quad 9: 36: 43$ & 554 & 4632 & 26.3125 & 26. 25 1590BE83 & CH1: & 1525 & 1525 & 1525 & 1525 & 1525 & 1525 \\
\hline 36088 & $2019-12-6 \quad 9: 36: 43$ & 604 & 4633 & 26.3125 & 26. 25 19E0BE83 & CH1: & 1525 & 1525 & 1525 & 1525 & 1525 & 1525 \\
\hline 36089 & $2019-12-6 \quad 9: 36: 43$ & 654 & 4634 & 26.3125 & 26. $25 \mathrm{OBF} 83$ & CH1: & 1525 & 1525 & 1525 & 1525 & 1525 & 1525 \\
\hline 36090 & $2019-12-6 \quad 10: 04: 18$ & 432 & 4635 & 28.4375 & $28.75450 \mathrm{BF} 83$ & CH1: & 1525 & 1525 & 1525 & 1525 & 1525 & 1525 \\
\hline 36091 & $2019-12-6 \quad 10: 04: 18$ & 528 & 4636 & 28.4375 & 28. $758 \mathrm{AOBF} 83$ & CH1: & 1525 & 1525 & 1525 & 1525 & 1525 & 1525 \\
\hline 36092 & $2019-12-6 \quad 10: 04: 19$ & 840 & 4637 & 28.4375 & 28. $75 \mathrm{CF} 0 \mathrm{BF} 83$ & CH1: & 1525 & 1525 & 1525 & 1525 & 1525 & 1525 \\
\hline 36093 & $2019-12-6 \quad 14: 46: 40$ & 964 & 4702 & 28.4375 & $28.81251140 \mathrm{BF} 83$ & $\mathrm{CH} 1$ : & & & & & & \\
\hline 36094 & $2019-12-6 \quad 14: 46: 41$ & 14 & 4703 & 28.4375 & $28.81251590 \mathrm{BF} 83$ & $\mathrm{CH} 1:$ & & & & & & \\
\hline 36095 & $2019-12-6 \quad 14: 46: 41$ & 64 & 4704 & 28.4375 & 28. $812519 \mathrm{E} 0 \mathrm{BF} 83$ & CH1: & & & & & & \\
\hline 36096 & $2019-12-6 \quad 14: 46: 41$ & 114 & 4705 & 28.4375 & $28.81250 \mathrm{C} 083$ & CH1: & & & & & & \\
\hline 36097 & $2019-12-6 \quad 14: 46: 41$ & 164 & 4706 & 28.4375 & $28.8125450 \mathrm{C} 083$ & CH1: & & & & & & \\
\hline
\end{tabular}

图4 上电一段时间后从异常恢复正常数据。

\section{2. 原因分析}

通过观察存储的时间信息, 可以看出月和日均为 0 的情 况，但通过进行代码走查判断，该情况在逻辑中不应该出 现，因软件在初始化阶段即对月和日变量进行了初始化 (1 月1日），且在软件运行过程中完全不存在清零的过程，除 非在时间同步时输入了错误的时间信息，但由于时间输入 是通过读取计算机系统时间的方式进行，不会出现日和月
为 0 的情况。通过数据产生的可能性分析：可能是因为两组 数据耦合，导致时间信息数据异常（NAND-flash特性为数 据耦合可以使得存储的数据位从 1 变为 0 , 但不能从 0 变成 1 , 耦合方式可以认为两组数据进行了相与 (\&) 操作)。

表 1 是对帧计数进行上下两次通电的数据耦合分析, 可以看出从第 1 帧到第 4701 帧均完美耦合，与存储的flash 数据完全一致。

表1 帧计数上下两次通电的数据耦合分析。

\begin{tabular}{|c|c|c|c|c|c|}
\hline 序号 & flash中帧计数 & 写入帧计数 & 写入帧计数 (16进制) & flash中帧计数（16进制） & 读取的帧计数 (16进制) \\
\hline & 229601 & 1 & 1 & $380 \mathrm{E} 1$ & 1 \\
\hline & 229602 & 2 & 2 & $380 \mathrm{E} 2$ & 2 \\
\hline & 229603 & 3 & 3 & $380 \mathrm{E} 3$ & 3 \\
\hline & 229604 & 4 & 4 & $380 \mathrm{E} 4$ & 4 \\
\hline & 229605 & 5 & 5 & $380 \mathrm{E} 5$ & 5 \\
\hline & 229606 & 6 & 6 & $380 \mathrm{E} 6$ & 6 \\
\hline & 229607 & 7 & 7 & $380 \mathrm{E} 7$ & 7 \\
\hline & 229608 & 8 & 8 & $380 \mathrm{E} 8$ & 8 \\
\hline & 229609 & 9 & 9 & $380 \mathrm{E} 9$ & 9 \\
\hline & 229610 & 10 & A & $380 \mathrm{EA}$ & A \\
\hline & 229611 & 11 & B & $380 \mathrm{~EB}$ & B \\
\hline & 229612 & 12 & $\mathrm{C}$ & $380 \mathrm{EC}$ & $\mathrm{C}$ \\
\hline & 229613 & 13 & D & $380 \mathrm{ED}$ & D \\
\hline & 229614 & 14 & E & $380 \mathrm{EE}$ & E \\
\hline & 229615 & 15 & $\mathrm{~F}$ & $380 \mathrm{EF}$ & $\mathrm{F}$ \\
\hline & 229616 & 16 & 10 & $380 \mathrm{~F} 0$ & 10 \\
\hline & 229617 & 17 & 11 & $380 \mathrm{~F} 1$ & 11 \\
\hline & 229618 & 18 & 12 & $380 \mathrm{~F} 2$ & 12 \\
\hline & 229619 & 19 & 13 & $380 \mathrm{~F} 3$ & 13 \\
\hline & 229620 & 20 & 14 & $380 \mathrm{~F} 4$ & 14 \\
\hline
\end{tabular}




\begin{tabular}{llllll}
\hline 序号 & flash中帧计数 & 写入帧计数 & 写入帧计数 (16进制) & flash中帧计数 (16进制) & 读取的帧计数 (16进制) \\
\hline & 229621 & 21 & 15 & $380 \mathrm{~F} 5$ & 15 \\
& 229622 & 22 & 16 & $380 \mathrm{~F} 6$ & 16 \\
& 229623 & 23 & 17 & $380 \mathrm{~F} 7$ & 17 \\
& 229624 & 24 & 18 & $380 \mathrm{~F} 8$ & 18 \\
& 229625 & 25 & 19 & $380 \mathrm{~F} 9$ & $1 \mathrm{~A}$ \\
& 229626 & 26 & $1 \mathrm{~A}$ & $380 \mathrm{FA}$ & $1 \mathrm{~B}$ \\
& 229627 & 27 & $1 \mathrm{~B}$ & $380 \mathrm{FB}$ & $1 \mathrm{C}$ \\
& 229628 & 28 & $1 \mathrm{C}$ & $380 \mathrm{FC}$ & $1 \mathrm{D}$ \\
& 229629 & 29 & $1 \mathrm{D}$ & $380 \mathrm{FD}$ & $1 \mathrm{~F}$ \\
& 229630 & 30 & $1 \mathrm{E}$ & $380 \mathrm{FE}$ & 0 \\
& 229631 & 31 & $1 \mathrm{~F}$ & $380 \mathrm{FF}$ & 1 \\
& 229632 & 32 & 20 & 38100 & $\ldots \ldots$ \\
$\ldots$ & 229633 & 33 & 21 & $\ldots \ldots$. & $121 \mathrm{D}$ \\
\hline
\end{tabular}

通过对时间信息和帧数据信息进行数据分析, 可以得 出以下结论: 数据异常故障的原因是由于上下两次上电存 储的部分数据进行耦合的原因导致的。该区域flash块在上 一次断电前已写数, 系统重新上电后, 该区域又被重新写 入数据, 导致前后两次数据进行了耦合。

系统在进行可靠性设计时, 已充分考虑了多次上电可 能导致的块地址中数据重复写入导致数据的不正确性的 情况, 且在下一次上电后自动隔离一个存储周期的地址长 度, 保证了NAND-flash存储器中数据存储的圥余。
通过新增代码, 将存入到块地址存储区中的addr1 和addr2实时读出并通过串口往外发送, 保存数据进行 分析后发现, addr1和addr2数据未按照设计要求的一个 存储周期的地址长度递增, 与上述存储的温度/应变数 据出现的情况类似, 出现了乱码的情况, 同样可以发现 该数据是由于前后连续两次存储的数据进行耦合的原 因导致的。

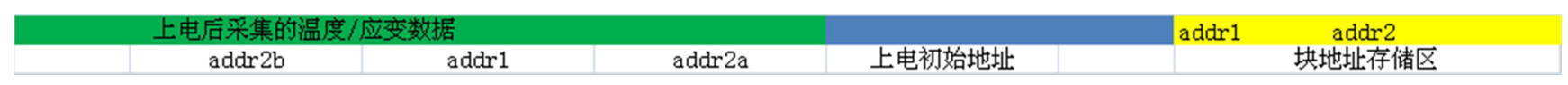

图5 地址数据。

由于 addr 1 和addr2数据的错误, 可以根据图 3 进行以下 分析, addr2a表示 $\mathrm{addr} 1$ 下一个存储的地址数据, $\operatorname{addr} 2 \mathrm{~b}$ 表 示addr1 上一个存储的地址数据, addr2a和 $\mathrm{addr} 2 \mathrm{~b}$ 均存于块 地址的addr2区域, 对addr2b擦除后, 存入add2a, 同时他们 之间的关系为:

$$
\begin{aligned}
& \operatorname{addr} 2 \mathrm{a}=\operatorname{addr} 1+\text { 一个存储周期的地址长度 } \\
& \operatorname{addr} 2 \mathrm{~b}=\mathrm{addr} 1 \text { - 一个存储周期的地址长度 }
\end{aligned}
$$

由于 $a d d r 2 b$ 与 $a d d r 2 a$ 数据耦合, 耦合得到的数据 $\mathrm{addr} 2<\mathrm{addr} 2 \mathrm{~b}<\mathrm{addr} 1$ （flash数据耦合可以使得存储的数据 位从 1 变为 0 , 但不能从 0 变成 1$)$, 故而导致原逻辑判断下 一次上电的初始块地址变成addr1+一个存储周期的地址 长度, 与设计期望的addr $2 \mathrm{a}+$ 一个存储周期的地址长度少 了一个存储周期的地址长度, 导致存储的部分温度/应变 数据耦合。

\section{4. 解决措施及试验验证}

为了避免出现存储的温度/应变数据的异常故障的出 现, 关键在于解决前后两次连续通电的数据耦合问题, 也 就是解决上电初始块地址的正确性问题。

针对上述问题, 主要提出了两种解决措施。第一种, 增加NAND-flash块地址存储区存储数据前进行擦除的等 待时间, 由原芯片手册中推荐使用的 typicaltime改为 maxtime, 保证具有充分的时间将存储区中的内容擦除。 第二种, 保证块地址存储区写入数据的正确性校验功能,
数据地址写入后立即读出, 与写入数据进行比较, 如数据 不一致, 重新进行写入操作, 以上循环进行 2 3 次, 充分 保证写入数据的正确性。

为了进一步增加NAND-flash温度/应变数据存储的可 靠性, 在系统上电确定初始块地址后, 从初始块地址中读 取数据, 判断初始化块地址是否为空或擦除状态 (判断数 据是否为 $0 x f f$ ), 如不为空和擦除状态, 则初始块地址往 后延一个固定长度, 重新判断初始块地址的状态, 以此循 环, 直至初始块地址为擦除状态。

将改进后的系统多次上电, 并将存入到块地址存储区 中的 $\mathrm{addr} 1$ 和addr 2 实时读出并通过串口往外发送, 进行数 据分析后发现 $\mathrm{addr} 1$ 和addr2数据按照设计要求的一个存储 周期的地址长度递增, 不再出现数据异常情况, 同时, NAND-flash存储的温度/应变数据也未出现数据异常情况, 该问题得到解决。

\section{5. 结论}

凭借着存储密度大和存储速率高的特点, 基于 NAND-flash的大容量存储器在航天型号领域得到了广泛 的应用。但是由于NAND-flash本身存在缺陷, 包括坏块、 擦除和写入异常等情况, 依靠NAND-flash本身无法有效保 证航天型号数据存储的高可靠性要求。本文针对航天型号 温度/应变测量系统中出现的数据异常情况, 分析了基于 NAND-flash的数据存储异常故障机理及解决措施并进行 了试验验证。该方法在航天型号领域大容量、高可靠性数 据存储方面具有较高的参考意义。 


\section{参考文献}

[1] 乔立岩, 李元亨, 王戈, 等.一种NAND Flash坏块管理优化 方法的实现 $[\mathrm{J}]$, 电子测量技术, 2015.07。

[2] 彭兵, 步凯, 徐欣, NAND Flash坏块管理研究 [J], 微处理 机, 2009.02。

[3] 周军, NAND Flash的坏块管理设计 $[\mathrm{J}]$, 单片机与嵌入式系 统应用, 2010.09。

[4] 舒文丽, 吴云峰, 赵启义, 孙长胜, NAND Flash存储的坏 块管理方法 $[\mathrm{J}]$, 电子器件, 2011.05。

[5] Samsung, datasheet: 256MX8Bit/64MX16BitNAND Flashmemory [S], Samsung Electronics, Revision 0.2, March 2003.

[6] Samsung. NAND Flash spare assignment recommendation [S], http//wwn.Sumsung.corn,2003.

[7] 蔡锦达, 王德福, 大容量Nand Flash在ARM嵌人式系统中 的应用 $[\mathrm{J}]$, 工业控制计算机.2004,06。

[8] 谢民, 高梅国, 王超, NAND型Flash在大容量存储回放系 统中的应用 $[\mathrm{J}]$, 电子技术应用, 2006.4。

[9] 高文青, 沈维聪, NAND存储器在嵌入式系统中的应用 $[\mathrm{J}]$, 电测与仪表, 2008.02。

[10] 高怡祯, 基于闪存的星载大容量存储器的研究和实现 $[\mathrm{J}]$, 集成电路应用2003.08。

[11] 张伟功, 邱庆林, 荣金叶, 高可靠性大容量星载存储器的 设计与研究 [J],第三次会议, 2010.08。

[12] 李进, 金龙旭, 韩双丽, 李国宁, 王文华, 间图像存储器 NAND Flash的可靠性 $[\mathrm{J}]$, 光学精密工程, 2012.05。

[13] 沈浩, 付宇卓, NAND Flash存储控制器的软硬件划分设计 [J], 计算机工程, 2004.24。 